ARTICLE

Received 22 Jan 2013 | Accepted 18 Apr 2013 | Published 28 May $2013 \quad$ DOl: 10.1038/ncomms2911

\title{
Non-equilibrium nature of two-dimensional isotropic and nematic coexistence in amyloid fibrils at liquid interfaces
}

\author{
Sophia Jordens ${ }^{1}$, Lucio Isa², Ivan Usov \& Raffaele Mezzenga ${ }^{1}$
}

Two-dimensional alignment of shape-anisotropic colloids is ubiquitous in nature, ranging from interfacial virus assembly to amyloid plaque formation. The principles governing two-dimensional self-assembly have therefore long been studied, both theoretically and experimentally, leading, however, to diverging fundamental interpretations on the nature of the two-dimensional isotropic-nematic phase transition. Here we employ single-molecule atomic force microscopy, cryogenic scanning electron microscopy and passive probe particle tracking to study the adsorption and liquid crystalline ordering of semiflexible $\beta$-lactoglobulin fibrils at liquid interfaces. Fibrillar rigidity changes on increasing interfacial density, with a maximum caused by alignment and a subsequent decrease stemming from crowding and domain bending. Coexistence of nematic and isotropic regions is resolved and quantified by a length scale-dependent order parameter $S_{2 D}(d)$. The nematic surface fraction increases with interfacial fibril density, but depends, for a fixed interfacial density, on the initial bulk concentration, ascribing the observed two-dimensional isotropic-nematic coexistence to non-equilibrium phenomena.

\footnotetext{
${ }^{1}$ ETH Zurich, Department of Health Sciences and Technology, Laboratory of Food \& Soft Materials, Schmelzbergstrasse 9, 8092 Zurich, Switzerland. ${ }^{2}$ ETH Zurich, Department of Materials, Laboratory for Surface Science \& Technology, Wolfgang-Pauli-Strasse 10, 8093 Zurich, Switzerland. Correspondence and requests for materials should be addressed to R.M. (email: raffaele.mezzenga@hest.ethz.ch).
} 
iquid crystalline (LC) ordering of rigid macromolecules, biopolymers and colloids is a widespread phenomenon in nature. In three dimensions (3Ds), the isotropicnematic (IN) phase transition is a well understood first-order transition ${ }^{1-3}$, characterized by a density range where both domain types coexist. The nematic phase is stabilized by excluded volume effects: The translational entropy gained by alignment exceeds the orientational entropy lost, thus reducing the total free energy ${ }^{1}$. LC alignment can also occur in two dimensions (2Ds) via interfacial adsorption and confinement. Of particular interest is the self-organization of macromolecules with a high aspect ratio into LC assemblies at interfaces, which has been treated theoretically ${ }^{4-6}$ but less frequently shown experimentally ${ }^{7,8}$. In contrast to the $3 \mathrm{D}$ case and under the assumption of thermodynamic equilibrium, the $2 \mathrm{D}$ IN transition can be of first ${ }^{4}$, second order ${ }^{5}$, or of the Kosterlitz-Thouless continuous type leading to quasi-long-range order ${ }^{9-11}$, depending on the interactions between particles ${ }^{12}$ and their rigidity ${ }^{10}$. Theoretical evidence for a truly $2 \mathrm{D}$ second-order transition is based on mean-field assumptions, and therefore does not account for fluctuations, which can be responsible for the suppression of long-range nematic order. Finally, most of the available studies rely on rigid particles; theoretical or simulation studies on semiflexible particles, in particular, are still very limited ${ }^{6,10}$ and practically non-existent in conditions far from thermodynamic equilibrium.

Alignment of highly anisotropic nano- or microparticles at interfaces is typically achieved experimentally either by compression in a Langmuir trough, usually by spreading the particles on the surface to circumvent adsorption from the subphase ${ }^{13,14}$, or through Langmuir-Blodgett deposition ${ }^{15,16}$ on prepatterned solid substrates to improve alignment ${ }^{17,18}$. $2 \mathrm{D}$ liquid crystallinity in drying droplets of semiflexible biopolymers such as DNA or F-actin solutions has also been reported ${ }^{19,20}$. The efforts made to understand the microstructure of anisotropic particle-laden interfaces demonstrate high fundamental interest. In the case of amyloid fibrils, in particular, three main areas of research are noteworthy ${ }^{21}$. First and foremost, their accumulation at biological interfaces is a crucial step in pathological processes such as plaque/deposit formation ${ }^{22}$ in membrane-associated amyloid diseases such as Alzheimer's or type II diabetes mellitus ${ }^{23}$. Second, several naturally occurring functional amyloids are known to predominantly act at interfaces: curli fibrils facilitate the formation of enterobacteria biofilm $\mathrm{s}^{24,25}$ and hydrophobins assemble into amyloid-like rodlets at the air-water interface to direct aerial hyphae growth of fungi ${ }^{26}$. Third, protein fibrils are attractive candidates to exploit the vast range of possible technological applications of surface-active nanoparticles for scaffolding ${ }^{27}$ or biocompatible capsules ${ }^{28}$.

We present experimental evidence from three distinct and independent techniques for adsorption-governed 2D LC ordering of highly anisotropic semiflexible amyloid fibrils at air-water and oil-water interfaces, where nematic and isotropic domains coexist. Passive probe particle tracking, atomic force microscopy (AFM), and freeze-fracture shadow-casting cryogenic scanning electron microscopy (FreSCa cryo-SEM) ${ }^{29}$ concurrently show that nematic fibril domains form upon increase in interfacial density $\rho$. We follow the evolution of LC ordering in the absence of an external compressive force, as opposed to the majority of previous experimental works. The apparent flexibility of fibrils adsorbed at the interface changes as a function of alignment and crowding. We evaluate this alignment with a length scale-dependent $2 \mathrm{D}$ order parameter $S_{2 \mathrm{D}}(d)$ and determine the amount of nematic coverage at the interface, which is found to not only depend on the local density but also the initial bulk fibril concentration $c_{\text {init }}$, pointing at an out-of-equilibrium ordering process.

\section{Results}

Passive probe particle tracking. As shown in our previous work ${ }^{8}$, passive fluorescent particle tracking can shed light on the interface microstructure. Using the same experimental protocol (see Methods section and Supplementary Note 1) and fluorescent silica tracers (diameter $=774 \pm 90 \mathrm{~nm}$ ), we built a tracer anisotropy map of an oil-aqueous fibril solution $\left(c_{\text {init }}=0.01 \%\right.$ $\mathrm{w} / \mathrm{w})$ interface after $45 \mathrm{~min}$, which shows striking spatial heterogeneity (Fig. 1a). At high concentrations (albeit much lower than in bulk systems $\mathrm{s}^{30,31}$ ), there is a coexistence of regions of highly anisotropic and isotropic tracer motion, indicative of the coexistence of aligned and randomly oriented fibril domains. For those tracers which are forced along the local director of fibril alignment, the principal direction of anisotropic motion is indicated by a blue line, and we can immediately see that these lines are not aligned over the whole field of view. This indicates that, in addition to the fact that fibrils form nematic islands at the interface, the directors of these domains are, as well, not aligned over the entire interfacial area.

Evolution of 2D structure revealed by AFM and cryo-SEM. To further illuminate the dynamic process of fibrillar alignment at liquid interfaces, original, undisturbed air-water interfacial layers of fibrils at different adsorption times were imaged by dry state AFM after transfer onto atomically flat mica by a modified Langmuir-Schaefer method. Briefly, a freshly cleaved mica sheet was brought into contact horizontally with the uncompressed liquid sample surface. Excess water on the mica after lift-off was exchanged with ethanol to remove unbound fibrils in the bulk ${ }^{32}$. More details and evidence for the artefact-free nature of the transfer are given in the Methods section, Supplementary Fig. S1 and Supplementary Note 2. Closely packed domains of as many as 20 highly aligned fibrils were found on areas spanning several hundreds of $\mu \mathrm{m}^{2}$ indicative of quasi-long-range order (Fig. 1b). The collective bending of fibrils in individual domains (Fig. 1c) is a consequence of the high interfacial density as discussed later. FreSCa cryo-SEM imaging validates that the aligned fibrils visible in AFM images are in fact the topmost fibrils at the interface and that alignment is not due to sample preparation (Fig. 1d). Here, the images are taken from the oil side after fracture showing only the surface of the vitrified water where the alignment of fibrils exhibits remarkable similarities with that seen in AFM images. The slightly larger apparent fibril diameter in SEM (height = $2-10 \mathrm{~nm}$ from AFM, width $=12-20 \mathrm{~nm}$ from SEM) can be explained by a combination of the deposited layer of tungsten and the presence of a hydration shell around the fibrils.

Figure 2 illustrates the time evolution of the adsorption at an air-water interface from a solution with $c_{\text {init }}=0.005 \% \mathrm{w} / \mathrm{w}$ studied by AFM. Starting with a sparsely covered surface at $t=10 \mathrm{~min}$, where fibrils are distributed mostly randomly (Fig. 2a), the surface density rapidly increases (Fig. 2b, $t=30 \mathrm{~min}$ and Supplementary Fig. S2), leading to the formation of patches of strongly aligned fibrils (Fig. 2c). Interspersed are areas with randomly oriented fibrils, hinting at a spindle-like tactoidal arrangement of nematic domains with homogeneous directors in an isotropic background, highlighting the importance of LC interactions, and which, from a thermodynamic point of view, would have the important consequence of identifying the observed $2 \mathrm{D}$ IN transition as a first-order transition. Owing to the random nature of nucleation and propagation of ordered domains and the highly dilute bulk concentration, the directors of nematic domains do not align macroscopically over the interface within the observed time frame ${ }^{33}$. Only at longer time and higher $c_{\text {init }}$ nematic domains may align along a common nematic director over areas as large as $10^{3} \mu \mathrm{m}^{2}$ (ref. 8). 
a

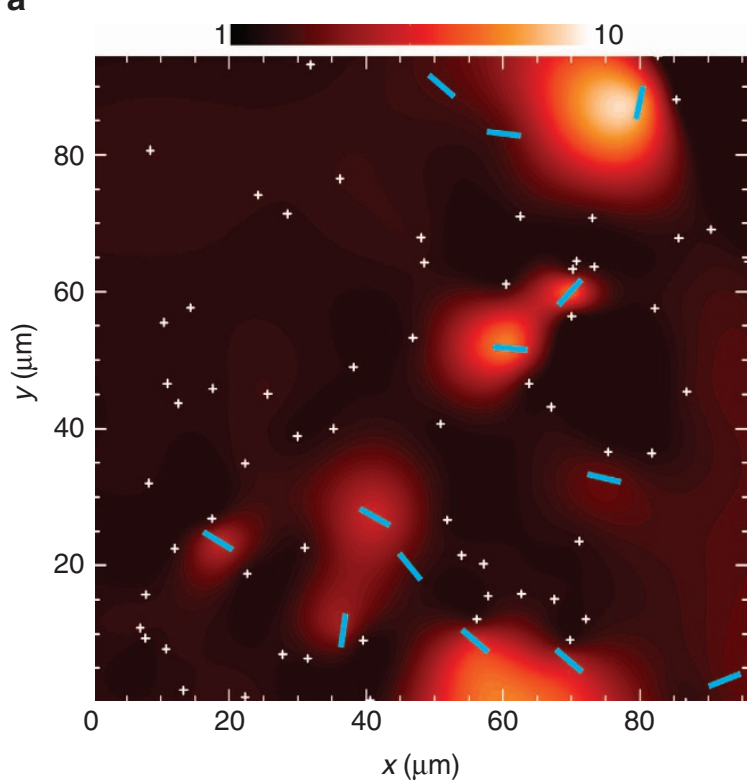

c

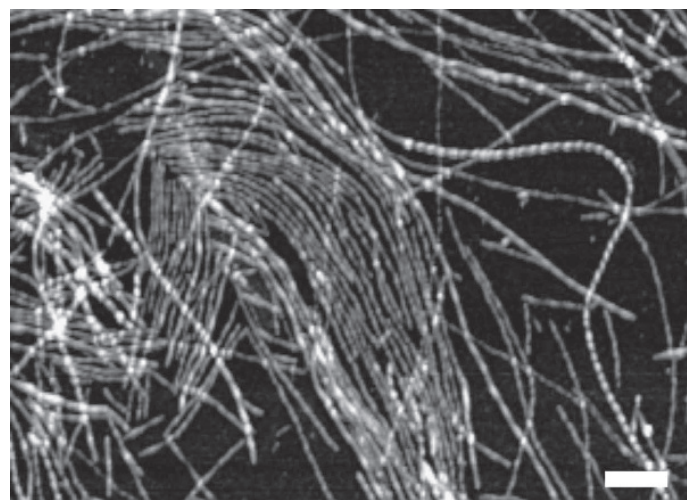

b

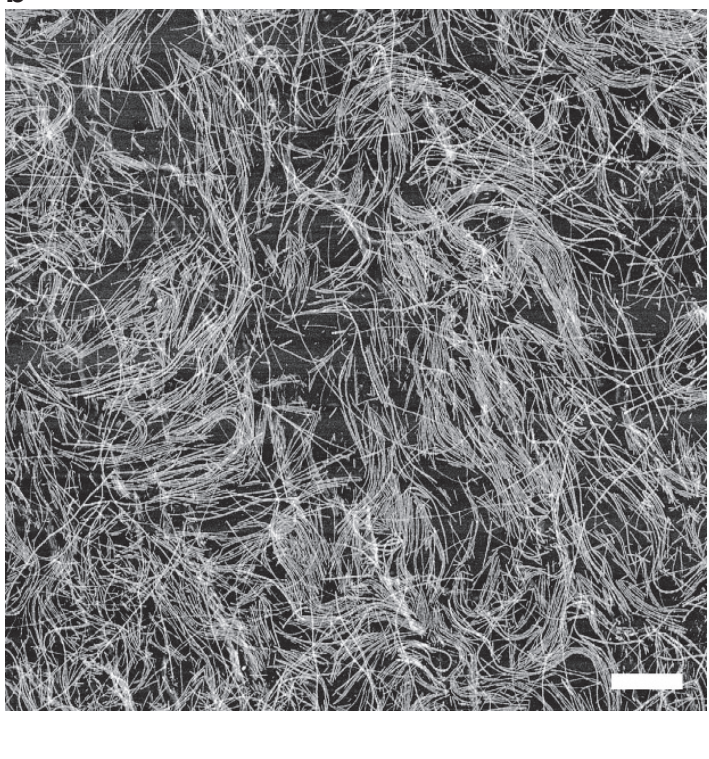

d

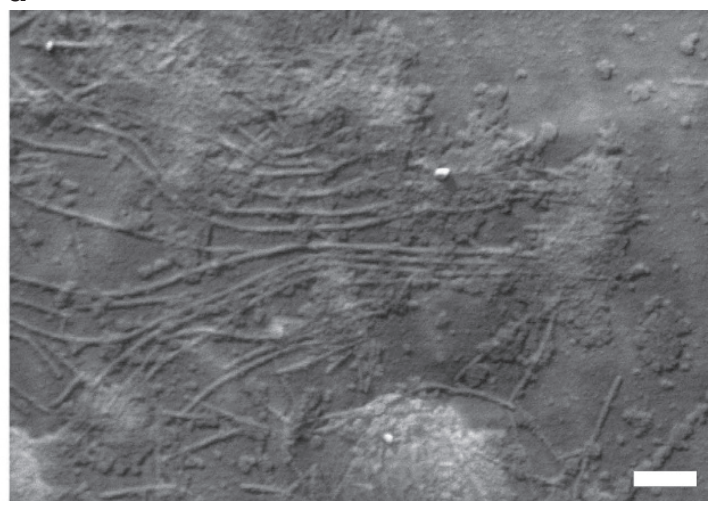

Figure 1 | Nematic alignment of $\boldsymbol{\beta}$-lactoglobulin fibrils at the liquid-liquid and gas-liquid interface. (a) Probe particle anisotropy map of an MCT-water interface at adsorption time $t=45 \mathrm{~min}$ and initial bulk concentration $c_{\text {init }}=0.01 \% \mathrm{w} / \mathrm{w}$. The average position of tracers is indicated by a white cross and for particles with highly anisotropic motion $\left(\left\langle\Delta x^{2}\right\rangle /\left\langle\Delta y^{2}\right\rangle>1.5\right)$ the director of motion is represented by a blue line. The red scale map represents local interpolated motion anisotropy. (b) AFM image of the air-water interfacial fibril layer at $t=5 \mathrm{~min}$ and $c_{\text {init }}=0.01 \% \mathrm{w} / \mathrm{w}$, obtained via the modified Langmuir-Schaefer protocol. The scale bar corresponds to $1 \mu \mathrm{m}$. (c) High magnification of $\mathbf{b}$. (d) High magnification FreSCa cryo-SEM image of the MCT-water interfacial fibril layer showing fibrils at the frozen water surface at $t=5 \mathrm{~min}$ and $c_{\text {init }}=0.01 \% \mathrm{w} / \mathrm{w}$. The scale bars in $\mathbf{c}$ and $\mathbf{d}$ correspond to $200 \mathrm{~nm}$.

a

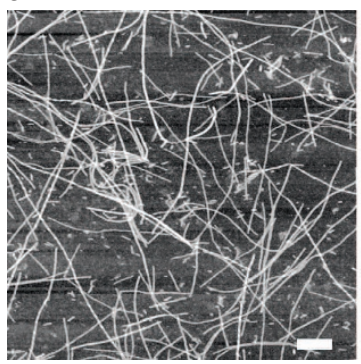

b

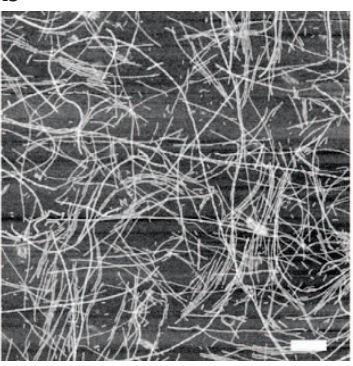

C

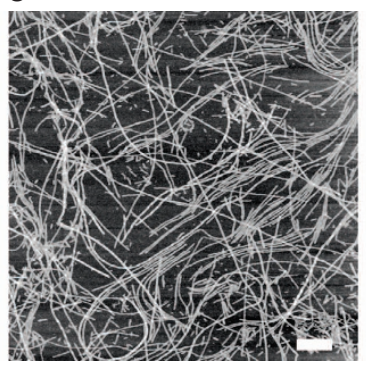

d

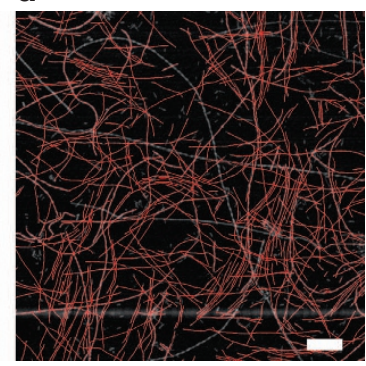

Figure 2 | Sequential AFM images of adsorption-controlled interfacial alignment of fibrils. As local fibril density at the air-water interface of a fibril solution with initial bulk concentration $c_{\text {init }}=0.005 \% \mathrm{w} / \mathrm{w}$ increases from (a) 10, (b) 30, until (c) 60 min, fibrils are more closely packed and start to form a two-dimensional liquid crystalline phase. (d) AFM image after tracking only fibrils in the topmost layer of the liquid interface (red) and excluding from statistical analysis those farthest away from the interface (white). The scale bars correspond to $500 \mathrm{~nm}$ in all images.

Liquid crystallinity and crowding affect bending length. To quantitatively assess the problem, we performed single- and collective-fibrils statistical analysis. The fibrils' contour $x y$ coordinates were extracted from AFM images by applying a custom-built tracking routine based on open active contours ${ }^{34}$. The persistence length $l_{\mathrm{p}}$ was calculated using the $2 \mathrm{D}$ worm-like 
chain model with the $\mathrm{fit}^{35}$ :

$$
\left\langle R_{2 \mathrm{D}}^{2}\right\rangle=4 L l_{\mathrm{p}}\left(1-\frac{2 l_{\mathrm{p}}}{L}\left(1-\exp \left(-\frac{L}{2 l_{\mathrm{p}}}\right)\right)\right),
$$

where $\left\langle R_{2 \mathrm{D}}^{2}\right\rangle$ is the average $2 \mathrm{D}$ mean square end-to-end distance and $L$ the internal contour length between any two points on a fibril. As $l_{\mathrm{p}}$ is a local, single-molecule property by definition and is not influenced by the interaction between fibrils (while the global quantity $\left\langle R_{2 \mathrm{D}}^{2}\right\rangle$ can indeed change), the term bending length $l_{\mathrm{b}}$ will be used instead of $l_{\mathrm{p}}$ to avoid confusion. Keeping in mind that what appears highest on the AFM images was farthest away from the liquid interface, we have intentionally discarded from the statistical analysis all fibrils that are clearly lying 'on top' of other fibrils to restrict the analysis to the topmost layer at the interface (Fig. 2d). Multilayer formation is energetically unfavourable because of the fibrils' charged nature and not observed in our experiments.

Early theoretical treatments by Khokhlov and Semenov ${ }^{36,37}$ formed the basis of the current understanding of the role played by flexibility in liquid crystallinity ${ }^{38}$. The opposite-how liquid crystallinity potentially affects particle flexibility-is much less understood $^{39,40}$. To the best of our knowledge, this effect has not yet been addressed in 2D, neither experimentally nor theoretically. Figure 3 shows the analysis of the $\rho$-dependent rigidity of fibrils adsorbing and crowding at liquid interfaces, where $\rho$ is the total interfacial density, expressed as contour length/unit area, with unit $\left(\mu \mathrm{m} \mu \mathrm{m}^{-2}=\mu \mathrm{m}^{-1}\right)$. Upon approaching each other and aligning into nematic domains, fibrils start to straighten to minimize orientational entropy. This results in an increase in $\left\langle R_{2 \mathrm{D}}^{2}\right\rangle$, and hence a twofold rise in rigidity from $l_{\mathrm{b}}=1.5$ to $3.0 \mu \mathrm{m}$. The $l_{\mathrm{b}}=1.5 \mu \mathrm{m}$ is in excellent agreement with previously reported $l_{\mathrm{b}}$ values of infinitely diluted $\beta$-lactoglobulin fibrils ${ }^{41}$, showing that interactions between fibrils are still negligible at the lowest density considered here (see Supplementary Fig. S3 for corresponding particle tracking data). As $\rho$ increases further and domains are more closely packed, whole domains are forced to undergo in-plane bending, reflected in a decrease in $l_{\mathrm{b}}$. The final $l_{\mathrm{b}}$ of fibrils in a crowded environment is $\sim 1.2 \mu \mathrm{m}$, slightly lower than the original, unperturbed $l_{\mathrm{b}}$ of $1.5 \mu \mathrm{m}$.

Length scale-dependent 2D order parameter. To quantify the local alignment, we introduce a length scale-dependent 2D order parameter $S_{2 \mathrm{D}}(d)$, where $d$ is the box size. This analysis is only meaningful well above a certain cutoff density $\rho_{0}$, namely, the density at which fibrils are no longer able to rotate freely, with rotational degrees of freedom controlled by excluded volume interactions ${ }^{1}$. The critical $\rho_{0}$ can be calculated solely based on $l_{\mathrm{p}}$, as the fibrils are semiflexible with full contour length $L_{\text {fibril }} \gg l_{\mathrm{p}}$ (refs 36, 37). Taking $\rho_{0}=l_{\mathrm{p}} /\left(\pi\left(l_{\mathrm{p}} / 2\right)^{2}\right)=4 /\left(\pi l_{\mathrm{p}}\right)$ and $l_{\mathrm{p}}=1.5 \mu \mathrm{m}$ (obtained at the lowest $\rho$ ), $\rho_{0}=0.85 \mu \mathrm{m}^{-1}$. All results presented in this work are based on densities above this threshold. The order of fibrils adsorbed on a given interface was quantified by considering them as sequences of very short, straight segments of equal length $(\sim 1$ pixel) connected along the fibril's contour, and applying the definition of the $2 \mathrm{D}$ LC order parameter, $S_{2 \mathrm{D}}=2\left\langle\cos ^{2} \theta\right\rangle-1$, to all segments contained in the interface of interest (see Methods section).

As there is a coexistence of strong alignment and completely randomly oriented fibrils on the same interface, the decay of the order parameter on each image $S_{2 \mathrm{D}}^{\text {image }}(d)$ is a sum of the weighted components $S_{2 \mathrm{D}}^{\mathrm{align}}$ and $S_{2 \mathrm{D}}^{\mathrm{ran}}$,

$$
S_{2 \mathrm{D}}^{\text {image }}(d)=a S_{2 \mathrm{D}}^{\text {align }}(d)+(1-a) S_{2 \mathrm{D}}^{\text {rand }}(d),
$$

where $a$ expresses the relative surface fraction of nematic domains and $S_{2 \mathrm{D}}^{\text {align }}(d)$ can be fitted with a single exponential decay function:

$$
S_{2 \mathrm{D}}^{\mathrm{align}}(d)=(1-b) \exp \left(-\frac{d}{2 \lambda}\right)+b .
$$

The offset $b$ accounts for the box size-dependent value of longrange order at infinite $d$ and the prefactor 2 in the exponent for the $2 \mathrm{D}$ nature of the experiment. The significance of $S_{2 \mathrm{D}}^{\text {rand }}$ originates from the fact that a purely isotropic state of vectors has an order parameter approaching zero only for a good enough statistic (that is, infinite box size). As $S_{2 D}^{\text {image }}$ is directly accessible from our statistical analysis algorithm applied to AFM images, we can extract information on the pure alignment component of fibrils $S_{2 \mathrm{D}}^{\text {align }}$, by calculating $S_{2 \mathrm{D}}^{\text {rand }}$ on a simulated image with fully randomized fibrils having identical $\rho, l_{\mathrm{b}}$ and $L_{\text {fibril }}$ distributions as in the image to be analysed (Fig. 4 and Methods section).

Figure 5a shows $S_{2 \mathrm{D}}^{\text {image }}$ and its decomposition into $S_{2 \mathrm{D}}^{\text {align }}$ and $S_{2 \mathrm{D}}^{\text {rand }}$ for an AFM image of fibrils at an air-water interface at $t=10 \mathrm{~min}$ with $c_{\text {init }}=0.005 \% \mathrm{w} / \mathrm{w}$ (Fig. 5a, bottom). Here, $a=0.06$ and therefore $\sim 94 \%$ of $S_{2 \mathrm{D}}^{\text {image }}$ is due to isotropic orientation of fibrils, while alignment has a negligible role at all
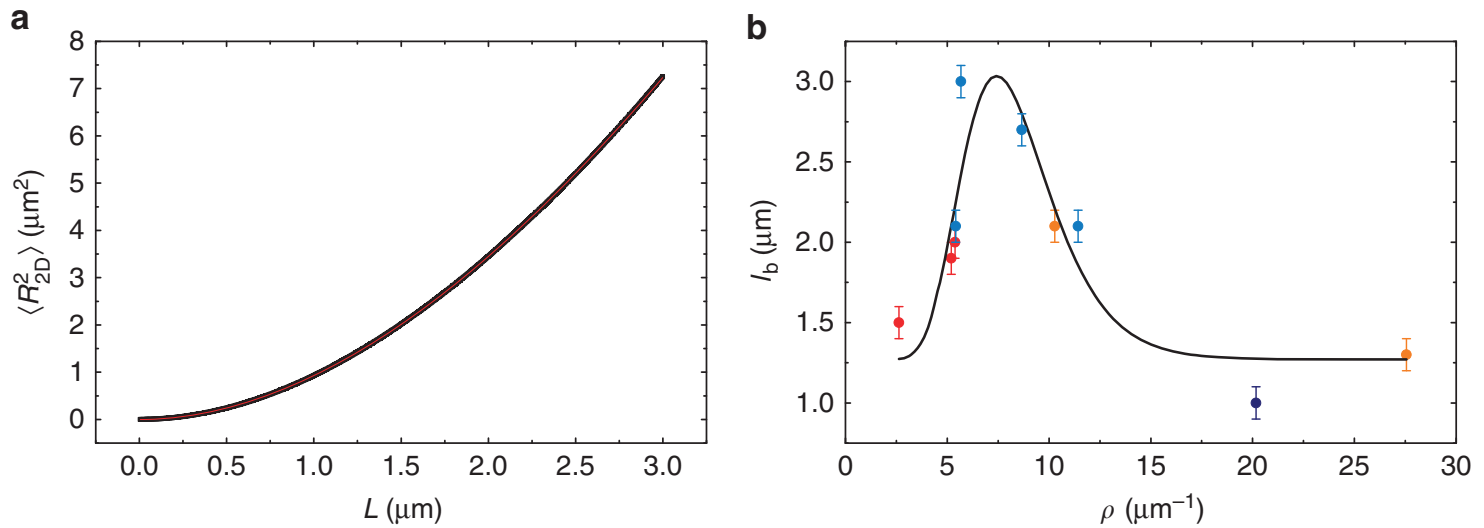

Figure 3 | Bending length of fibrils at an air-water interface. (a) Measured average two-dimensional mean square end-to-end distance $\left\langle R_{2 \mathrm{D}}^{2}\right\rangle$ versus internal contour length $L$. The fit for equation (1) yields persistence or bending length $I_{\mathrm{p}}$ or $I_{\mathrm{b}}$. Data shown correspond to fibrils at an air-water interface at adsorption time $t=30$ min with initial bulk concentration $c_{\text {init }}=0.005 \% \mathrm{w} / \mathrm{w}$, where $I_{\mathrm{b}}=2.1 \mu \mathrm{m}$. (b) $I_{\mathrm{b}}$ as a function of interfacial density $\rho$. Identical colours correspond to the same $c_{\text {init. }}$ Error bars represent the estimated accuracy of the fit. The solid line is a guide to the eye. 

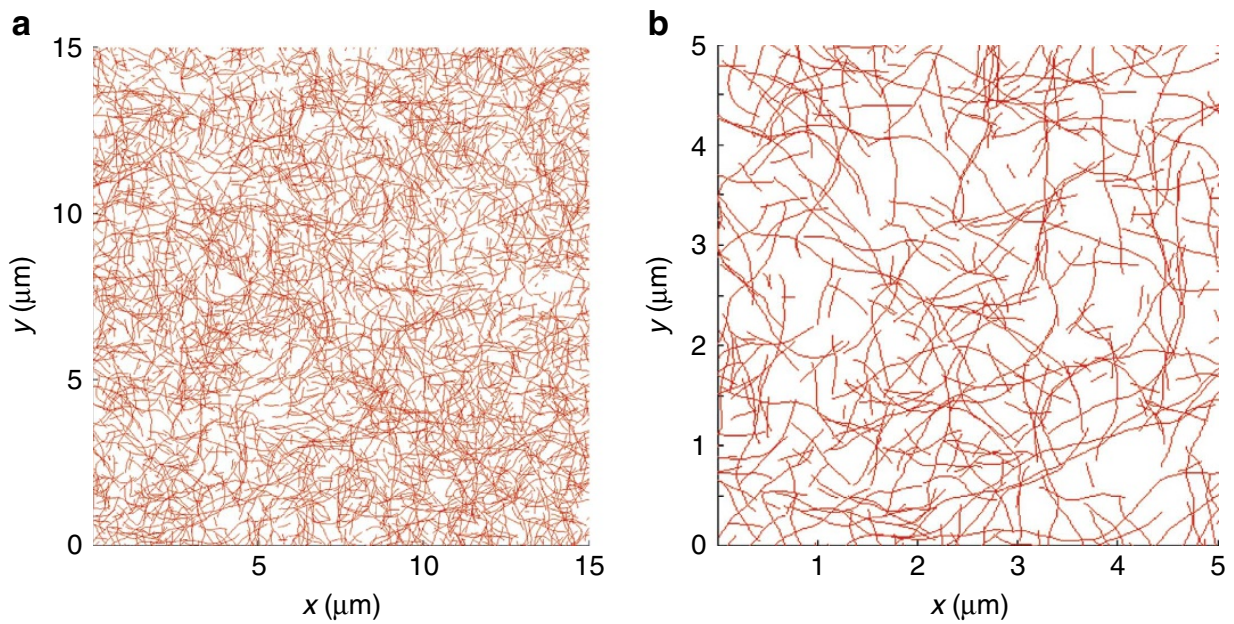

Figure 4 | Simulated image of fibrils. (a) Simulation and (b) magnification of the simulated image using parameters from an AFM image of the air-water interface at adsorption time $t=30 \mathrm{~min}$ with initial bulk concentration $c_{\text {init }}=0.005 \% \mathrm{w} / \mathrm{w}$ as input.

a
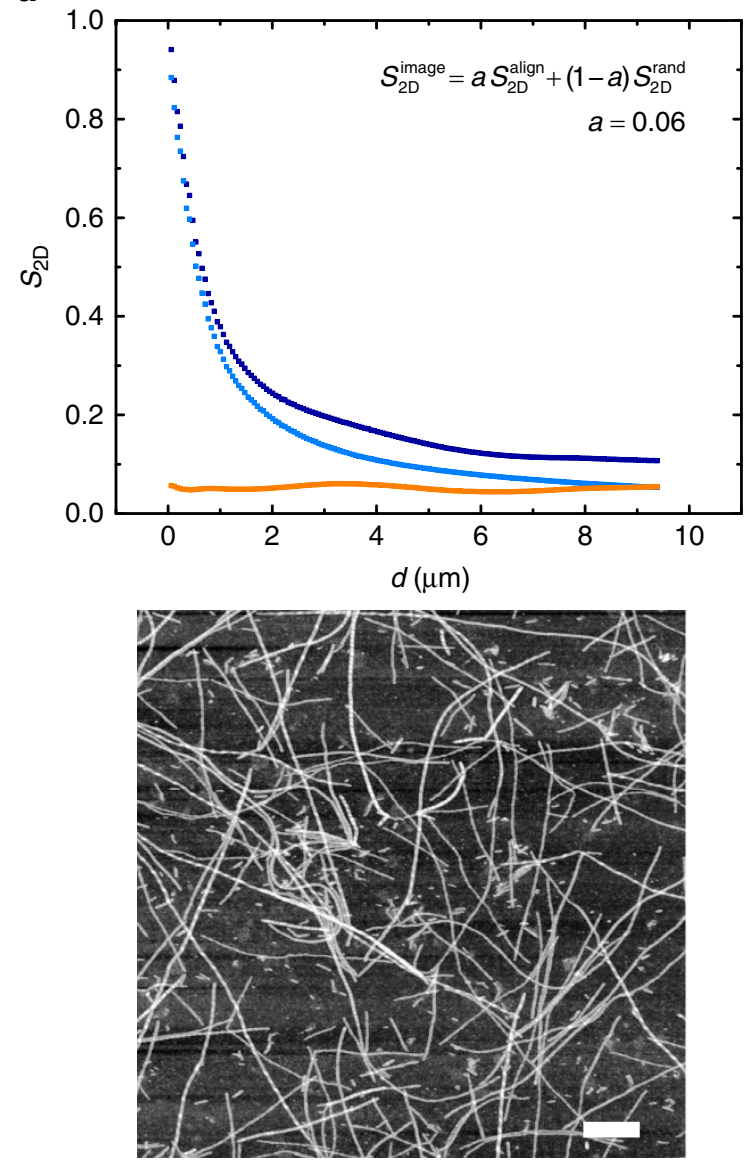

b
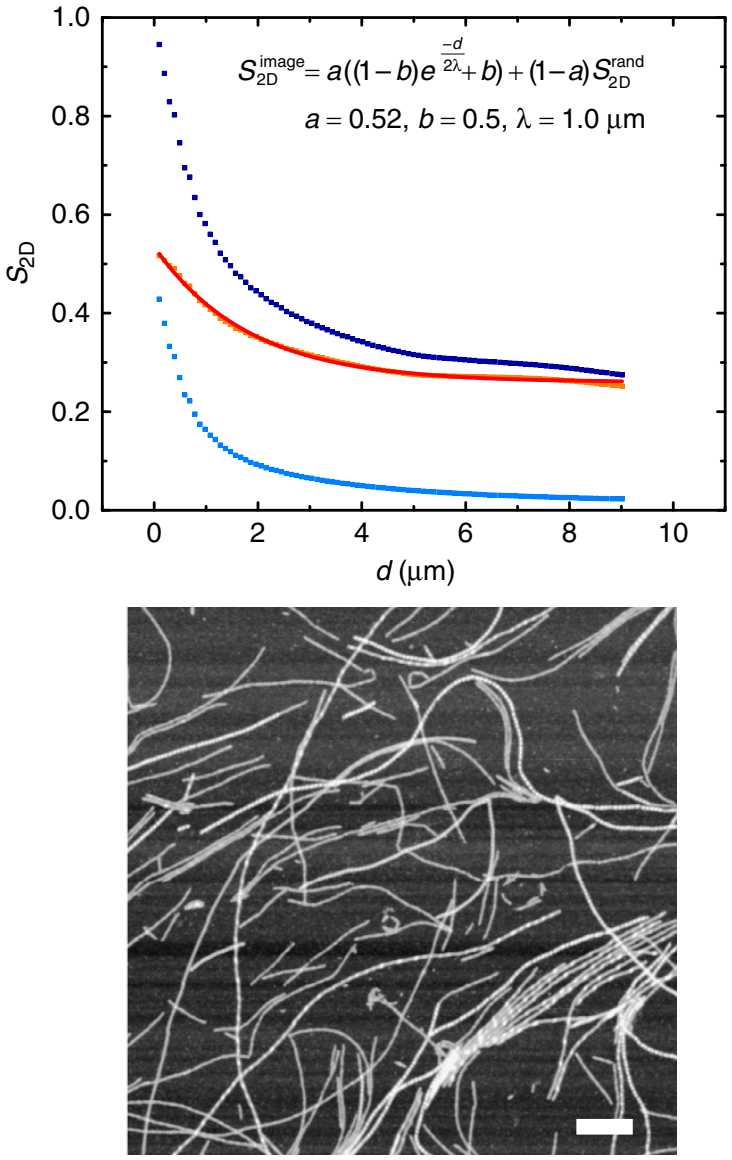

Figure 5 | Decomposition of $\boldsymbol{S}_{\mathbf{2 D}}^{\text {image }}(\boldsymbol{d})$ into $\boldsymbol{S}_{\mathbf{2 D}}^{\text {align }}$ and $\boldsymbol{S}_{\mathbf{2 D}}^{\text {rand }}$. The length scale-dependent 2D order parameter $S_{2 \mathrm{D}}^{\text {image }}(d)$ (dark blue), the weighted order parameter $(1-a) S_{2 D}^{\text {rand }}(d)$ on a corresponding simulated image (light blue), and the weighted difference $a S_{2 D}^{\text {align }}(d)$ (orange) for two images with similar interfacial densities $\rho \approx 5 \mu \mathrm{m}^{-1}$. (a) Adsorption time $t=10 \mathrm{~min}$, initial bulk concentration $c_{\text {init }}=0.005 \% \mathrm{w} / \mathrm{w}$. (b) $t=60 \mathrm{~min}, c_{\text {init }}=0.001 \% \mathrm{w} / \mathrm{w}$. $S_{2 D}^{\text {align }}(d)$ in $\mathbf{b}$ can be approximated with equation (3) and the fitting parameters $b=0.5$ and $\lambda=1.0 \mu \mathrm{m}$ (solid red line). Representative AFM images for both cases are shown in the bottom row. Both scale bars correspond to $500 \mathrm{~nm}$.

length scales. An accurate estimation of the $S_{2 \mathrm{D}}^{\text {align }}$ fitting parameters $\lambda$ and $b$ is impossible because of the low value of $a$. As expected and predictable from Fig. 2, $a$ increases with longer adsorption time. Intriguingly, however, not only the local density but also $c_{\text {init }}$ determines the overall order of the fibril layer. At a lower $c_{\text {init }}$ of $0.001 \% \mathrm{w} / \mathrm{w}$ it takes $60 \mathrm{~min}$ to 


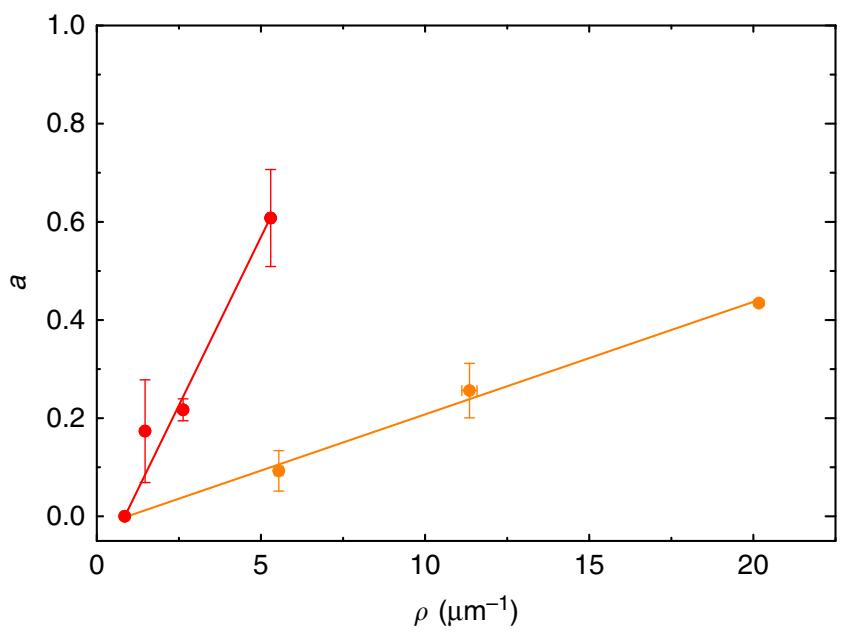

Figure 6 | The fraction of aligned fibrils depends on the initial bulk

concentration. Weighting factor $a$ as a function of interfacial density $\rho$ for initial bulk concentrations $c_{\text {init }}=0.001 \% \mathrm{w} / \mathrm{w}$ (red) and $c_{\text {init }}=0.005 \% \mathrm{w} / \mathrm{w}$ (orange). Error bars represent variations between different AFM images of the same sample. The solid lines are linear fits.

reach a similar density $\left(\rho \approx 5 \mu \mathrm{m}^{-1}\right)$, but at this concentration $\sim 52 \%$ of the interfacial area exhibits strong fibril alignment (Fig. 5b). $S_{2 \mathrm{D}}^{\text {align }}$ in this data set is well approximated by equation (3) with $b=0.5$ and $\lambda=1.0 \mu \mathrm{m}$. We propose that the length in the exponent is characteristic of the loss of order between individual nematic domains.

Dependence on initial bulk concentration. The present analysis offers the possibility to follow the evolution of the fraction of aligned fibrils $a$ as a function of $\rho$, as summarized in Fig. 6. The analysis clearly shows that higher alignment is observed for interfaces generated by adsorption from more dilute solutions. At a similar interfacial concentration $\left(\rho \approx 5 \mu \mathrm{m}^{-1}\right)$, the surface fraction of ordered fibrils in the adsorption experiment from dilute conditions is about six times that obtained by adsorption from a fivefold higher $c_{\text {init }}$.

\section{Discussion}

We used passive probe particle tracking, FreSCa cryo-SEM and AFM to consistently show that $\beta$-lactoglobulin amyloid fibrils align in $2 \mathrm{D}$ solely due to passive adsorption onto a liquid interface. Their end-to-end distance is shown to depend on the local density at the interface. Alignment causes fibrils to straighten, as reflected in an increase in $l_{\mathrm{b}}$, but as soon as the interfacial fibril coverage becomes so high that whole domains are forced to undergo in-plane bending, this value decreases again. This evolution of $l_{\mathrm{b}}$ in a crowded environment suggests that both excluded volume and pairwise interactions contribute to the change in fibril conformation.

We quantify the relative presence of isotropic and nematic interfacial regions by decomposing the total order parameter $S_{2 \mathrm{D}}^{\text {image }}$ into a length scale-dependent random and an aligned component, concluding that the nematic coverage strongly depends on the initial bulk concentration. This fact has the important implication that the state of interfacial alignment is not only a function of the total interfacial density, but also of the conditions by which this was achieved. A prerequisite for this must be a significant energy gain for the fibrils upon adsorption to the interface, which can be estimated by calculating the free energy of detachment $\Delta G_{\mathrm{d}}$ (ref. 42). Approximating the fibril with a long cylinder with aspect ratio $l / r=500$ (where $l=1,000 \mathrm{~nm}$ is half the length and $r=2 \mathrm{~nm}$ the radius) and contact angle $\theta_{\mathrm{c}}=90^{\circ}$ at the medium chain triglyceride (MCT)-water interface with interfacial tension $\gamma_{\mathrm{ow}}=30 \mathrm{~m} \mathrm{Nm}^{-1}$ (ref. 30), we get from

$$
\Delta G_{\mathrm{d}}=\gamma_{\mathrm{ow}} \pi r^{2}\left(1-\cos \theta_{\mathrm{c}}\right)^{2}\left(1+\frac{4\left(\frac{l}{r}-1\right)\left(\sin \theta_{\mathrm{c}}-\theta_{\mathrm{c}} \cos \theta_{\mathrm{c}}\right)}{\pi\left(1-\cos \theta_{\mathrm{c}}\right)^{2}}\right)
$$

a $\Delta G_{\mathrm{d}}$ of $\sim 6 \times 10^{4} \mathrm{kT}$. In view of the irreversibility of fibril adsorption, this immediately points to a non-equilibrium nature for the observed coexistence of $2 \mathrm{D}$ nematic and isotropic phases. A possible interpretation is that when adsorption takes place from low $c_{\text {init }}$, it proceeds at low enough rates to allow fibrils to align into defined and isolated 2D domains. This is supported by passive probe particle tracking data, showing that trace anisotropy occurs while tracer motion is still diffusive ${ }^{8}$. At higher $c_{\text {init }}$, the quenching rate is too high to permit unperturbed in-plane rotational rearrangement needed for the formation of nematic domains, leading to structures jammed into a more disordered alignment, hereby limiting the size of nematic domains, and yielding an overall viscoelastic interface (see Supplementary Fig. S4 and Supplementary Note 1). From the slopes of the linear fits in Fig. 6, we can estimate the rates of nematic domain formation versus interfacial concentration $\rho$, for two different bulk concentrations $c_{\text {init }}$, and find that the nematic fraction in the lower $c_{\text {init }}$ case grows six times faster than in the sample with fivefold higher $c_{\text {init }}$. We thus identify the phase coexistence as an out-of-equilibrium process. These findings bring new important elements to the understanding of complex anisotropic fluid interfaces. We see this work as a solid basis for the elucidation of interfacial self-organization processes where parameters such as charge, flexibility, polydispersity of colloidal object lengths and thicknesses, and even chirality may have a role.

\section{Methods}

Fibril sample preparation. Dialysed $\beta$-lactoglobulin fibrils were prepared as described previously ${ }^{30}$. All bulk concentrations are expressed in weight per cent as (weight of protein per weight of solution $\times 100$ ). Briefly, to purify the protein, a $10 \% \mathrm{w} / \mathrm{w} \beta$-lactoglobulin solution ( $\mathrm{pH} 4.6)$ was centrifuged at 15,000 r.p.m. for $15 \mathrm{~min}$. After collecting the supernatant and setting its $\mathrm{pH}$ to 2 , it was exposed to vacuum filtration through a $0.22 \mu \mathrm{m}$ cellulose filter and finally first dialysed against $\mathrm{pH} 2$ Milli-Q water and subsequently against Milli-Q water at $4{ }^{\circ} \mathrm{C}$ for $\sim 5$ days with twice daily and then daily bath changes to remove ions from the solution (Spectra/Por Dialysis Membrane 1, MWCO 6-8 kDa). After filtration through a $0.45 \mu \mathrm{m}$ cellulose filter and readjustment to $\mathrm{pH} 2$ and $2 \% \mathrm{w} / \mathrm{w}$, the solution was heated in a stirred oil bath at $90^{\circ} \mathrm{C}$ during $5 \mathrm{~h}$. The resulting $\beta$-lactoglobulin fibril solution was dialysed in Spectra/Por Biotech Cellulose Ester Dialysis tubes (MWCO $100 \mathrm{kDa}$ ) against $\mathrm{pH} 2$ Milli-Q water at $4{ }^{\circ} \mathrm{C}$ during 5 days with four bath changes to remove unconverted peptides. For experiments, the final fibril solution ( $\mathrm{pH} 2$, no salt) was varied by diluting a stock solution of $0.1 \% \mathrm{w} / \mathrm{w}$. MCT (Delios $\mathrm{GmbH}$ ) were used as the oil phase in all particle tracking and FreSCa cryo-SEM experiments.

Passive probe particle tracking. A well with a diameter of $1 \mathrm{~cm}$ was made out of ultraviolet-curable epoxy resin on a thoroughly cleaned glass cover slide using a patterned poly(dimethylsiloxane) stamp. Each microscopy cell was plasma-cleaned before the experiment to make the bottom of the well hydrophilic. The well was then filled with $15 \mu \mathrm{l}$ of aqueous sample solution seeded with $0.075 \% \mathrm{w} / \mathrm{v}$ positively charged fluorescent silica tracers (fluorescent dye: fluorescein isothiocyanate). Immediately after, MCT oil was carefully poured on top; some of the tracers were trapped at the flat oil-water interface and were imaged using an inverted microscope (Leica DMI6000B) with a $63 \times 1.4$ NA oil HCX PlanApo DIC objective. Up to 700 frames were grabbed at a rate of $0.374 \mathrm{~s}$ and the images were analysed with standard as well as custom-written software in IDL (ITT Visual Information Solutions $)^{43}$.

FreSCa cryo-SEM. Copper holders with a $200-\mu \mathrm{m}$-deep cavity were first cleaned in sulphuric acid (95\%) and ethanol, their surface was then roughened to improve adhesion during freezing, before finally being filled with $0.4 \mu \mathrm{l}$ aqueous fibril/tracer solution (the tracer particles were added to facilitate locating the interface during imaging). A drop of $3 \mu \mathrm{l} \mathrm{MCT}$ oil was carefully pipetted on top of the water surface before closing the holder with a copper plate and freezing the sample in a liquid propane jet freezer (Bal-Tec/Leica JFD 030, cooling rate $=30,000 \mathrm{~K} \mathrm{~s}^{-1}$ ). 
This extremely high rate vitrifies the water in the sample and no thermal expansion due to crystallization is observed. The holders were mounted onto a double fracture cryo-stage under liquid nitrogen and fractured under high vacuum at $-140^{\circ} \mathrm{C}$ (Bal-Tec/Leica BAF060). Partial freeze-drying at $-110^{\circ} \mathrm{C}$ for $3 \mathrm{~min}$ removed condensed water and ice crystals. After lowering the temperature to $-120^{\circ} \mathrm{C}$, a 2-nm thin layer of tungsten was deposited onto the sample surfaces at a $45^{\circ}$ angle to create a shadow behind each feature protruding from the vitrified interface and yield 3D information on the interface microstructure. A second 2-nm layer was subsequently deposited at constantly varying angles from 90 to $45^{\circ}$ to avoid charging of the shadow during imaging. Transfer to the precooled cryo-SEM (Zeiss Gemini 1530, Germany) was done under high vacuum $\left(<5 \times 10^{-7} \mathrm{mbar}\right)$ with an air-lock shuttle and the samples were imaged at $-120^{\circ} \mathrm{C}$ with a secondary electron detector.

Modified Langmuir-Schaefer technique. After dilution of the stock solution to the desired $c_{\text {init }}$, a glass vial (diameter $=2.5 \mathrm{~cm}$ ) was filled with $2 \mathrm{ml}$ sample solution avoiding the introduction of air bubbles. The vial was carefully covered to prevent evaporation and contamination of the liquid. Once the desired adsorption time had passed, a freshly cleaved mica sheet on a metal support was brought into horizontal contact with the sample surface and immediately removed again. Any excess glue around the mica had been thoroughly removed so as not to contaminate the sample. Mica was chosen as substrate because of its negative charge and almost step-free surface. Trials with graphite or silica showed that strong binding of the fibrils (positively charged) to the substrate is necessary for successful transfer. The mica was then dipped into ethanol $(\geq 99.8 \% \mathrm{v} / \mathrm{v})$ for phase exchange and removal of any unabsorbed material before slowly drying the sample under a weak clean air flow. Ethanol was chosen because its lower surface tension prevents the formation of artefacts at the receding contact line of the droplet during drying under a weak clean air flow, as shown for the case of non-densely packed 2D colloidal crystals ${ }^{32}$.

AFM imaging. AFM imaging was performed on a Nanoscope VIII Multimode Scanning Probe Microscope (Veeco Instruments) in tapping mode in air. Exact fibril tracking required image resolutions of $5,120 \times 5,120$ pixels for medium to high fibril density images on scan areas of $15 \times 15 \mu \mathrm{m}$ with a scan rate of $0.1 \mathrm{~Hz}$, while larger area scans were acquired whenever possible (lower density) to increase statistics.

2D order parameter algorithm. A square grid with constant cell edge $d_{0}$ (equal to the desired $x$ interval in the final graph) was applied to the image of interest. A lattice unit cell of area $d_{0}^{2}$ is the smallest area considered by the algorithm, which thus sets the resolution. $S_{2 \mathrm{D}}$ was calculated for all cells and averaged over the whole grid to produce the first data point $\left(d=d_{0}\right)$. Using the same grid as a basis, $S_{2 \mathrm{D}}$ for larger areas (with a step increment $d_{0}$ ) was calculated on the entire area covering all grid cells whose centers lie within $d / 2$ from the central point. These points were chosen as the centers of the circles inscribed in the corresponding squares of edge $d$. Translational shift vectors for the squares used for statistics were of the type $\left\{n_{1} d_{0} ; n_{2} d_{0}\right\}$, with $n_{1}$ and $n_{2}$ being integers.

Simulations. Simulated images of randomly oriented fibrils were created using all relevant parameters from the original images: mean $L_{\text {fibril }}$ from the peak of the fit of the log-normal $L_{\text {fibril }}$ distribution of all fibrils excluding those in contact with one of the borders of the image, the variance of the $L_{\text {fibril }}$ distribution, identical density $\rho$, $l_{\mathrm{b}}$ and segment length. The starting points and directions of objects were chosen randomly and filaments were then generated with a constant step size equal to the segment length used for tracking 35,44 . The simulation was run to produce very large images and a central crop of the desired size was used for calculations to avoid edge effects.

\section{References}

1. Onsager, L. The effects of shape on the interaction of colloidal particles. Ann. N. Y. Acad. Sci. 51, 627-659 (1949).

2. Saupe, A. Neuere Ergebnisse auf dem Gebiet der flüssigen Kristalle. Angew. Chem. 80, 99-115 (1968).

3. Flory, P. J. Phase equilibria in solution of rod-like particles. Proc. R. Soc. London A 234, 73-89 (1956).

4. Vink, R. L. C. Liquid crystals in two dimensions: first-order phase transitions and nonuniversal critical behavior. Phys. Rev. Lett. 98, 217801 (2007).

5. Marrucci, G. \& Maffettone, P. L. Description of the liquid-crystalline phase of rodlike polymers at high shear rates. Macromolecules 22, 4076-4082 (1989).

6. Chen, Z. Y. Continuous isotropic-nematic transition of partially flexible polymers in two dimensions. Phys. Rev. Lett. 71, 93-96 (1993).

7. Yoo, Z. Y. et al. Spontaneous assembly of viruses on multilayered polymer surfaces. Nat. Mater. 5, 234-240 (2006).
8. Isa, L., Jung, J. M. \& Mezzenga, R. Unravelling adsorption and alignment of amyloid fibrils at interfaces by probe particle tracking. Soft Matter 7, 8127-8134 (2011).

9. Bates, M. A. \& Frenkel, D. Phase behavior of two-dimensional hard rod fluids J. Chem. Phys. 112, 10034-10041 (2000).

10. Dijkstra, M. \& Frenkel, D. Simulation study of a two-dimensional system of semiflexible polymers. Phys. Rev. E 50, 349-357 (1994).

11. Lagomarsino, M. C., Dogterom, M. \& Dijkstra, M. Isotropic-nematic transition of long, thin, hard spherocylinders confined in a quasi-two-dimensional planar geometry. J. Chem. Phys. 119, 3535-3540 (2003).

12. Fish, J. M. \& Vink, R. L. C. Isotropic-to-nematic transition in confined liquid crystals: An essentially nonuniversal phenomenon. Phys. Rev. E 81, 021705 (2010).

13. Lepère, M., Chevallard, C., Brezesinski, G., Goldmann, M. \& Guenoun, P. Crystalline amyloid structures at interfaces. Angew. Chem. 121, 5105-5109 (2009).

14. Shortell, M. P., Liu, H.-W., Zhu, H., Jaatinen, E. A. \& Waclawik, E. R. Formation of one-dimensional capped $\mathrm{ZnO}$ nanoparticle assemblies at the air/ water interface. Langmuir 26, 14472-14478 (2010).

15. Kim, F., Kwan, S., Akana, J. \& Yang, P. Langmuir-Blodgett nanorod assembly. J. Am. Chem. Soc. 123, 4360-4361 (2001).

16. Tao, A. et al. Langmuir-Blodgett silver nanowire monolayers for molecular sensing using surface-enhanced Raman spectroscopy. Nano Lett. 3, 1229-1233 (2003).

17. Dorosz, J., Volinsky, R., Bazar, E., Kolusheva, S. \& Jelinek, R. Phospholipidinduced fibrillation of a prion amyloidogenic determinant at the air/water interface. Langmuir 25, 12501-12506 (2009).

18. Yang, L. et al. Structure and interaction in 2D assemblies of tobacco mosaic viruses. Soft Matter 5, 4951-4961 (2009).

19. Smalyukh, I. I., Zribi, O. V., Butler, J. C., Lavrentovich, O. D. \& Wong, G. C. L. Structure and dynamics of liquid crystalline pattern formation in drying droplets of DNA. Phys. Rev. Lett. 96, 177801 (2006).

20. Vonna, L., Limozin, L., Roth, A. \& Sackmann, E. Single-filament dynamics and long-range ordering of semiflexible biopolymers under flow and confinement. Langmuir 21, 9635-9643 (2005).

21. Cherny, I. \& Gazit, E. Amyloids: not only pathological agents but also ordered nanomaterials. Angew. Chem. Int. Ed. 47, 4062-4069 (2008).

22. Paparcone, R., Cranford, S. W. \& Buehler, M. J. Self-folding and aggregation of amyloid nanofibrils. Nanoscale 3, 1748-1755 (2011).

23. Selkoe, D. J. Folding proteins in fatal ways. Nature 426, 900-904 (2003).

24. Larsen, P. et al. Amyloid adhesins are abundant in natural biofilms. Environ. Microbiol. 9, 3077-3090 (2007).

25. Wu, C., Lim, J. Y., Fuller, G. G. \& Cegelski, L. Quantitative analysis of amyloidintegrated biofilms formed by uropathogenic Escherichia coli at the air-liquid interface. Biophys. J. 103, 464-471 (2012).

26. Gebbink, M. F. B. G., Claessen, D., Bouma, B., Dijkhuizen, L. \& Wösten, H. A B. Amyloids-a functional coat for microorganisms. Nat. Rev. Microbiol. 3, 333-341 (2005).

27. Liang, Y. et al. Light harvesting antenna on an amyloid scaffold. Chem. Commun. 2008, 6522-6524 (2008).

28. Humblet-Hua, K. N. P., Scheltens, G., van der Linden, E. \& Sagis, L. M. C. Encapsulation systems based on ovalbumin fibrils and high methoxyl pectin. Food Hydrocolloids 25, 569-576 (2011).

29. Isa, L., Lucas, F., Wepf, R. \& Reimhult, E. Measuring single-nanoparticle wetting properties by freeze-fracture shadow-casting cryo-scanning electron microscopy. Nat. Commun. 2, 438 (2011).

30. Jung, J. M. \& Mezzenga, R. Liquid crystalline phase behavior of protein fibers in water: experiments versus theory. Langmuir 26, 504-514 (2010).

31. Mezzenga, R., Jung, J. M. \& Adamcik, J. Effects of charge double layer and colloidal aggregation on the isotropic-nematic transition of protein fibers in water. Langmuir 26, 10401-10405 (2010).

32. Ray, M. A. \& Jia, L. Micropatterning by non-densely packed interfacial colloidal crystals. Adv. Mater. 19, 2020-2022 (2007)

33. Brand, H. R., Cladis, P. E. \& Pleiner, H. Symmetry and defects in the $C_{M}$ phase of polymeric liquid crystals. Macromolecules 25, 7223-7226 (1992).

34. Smith, M. B. et al. Segmentation and tracking of cytoskeletal filaments using open active contours. Cytoskeleton 67, 693-705 (2010).

35. Rivetti, C., Guthold, M. \& Bustamante, C. Scanning force microscopy of DNA deposited onto mica: equilibration versus kinetic trapping studied by statistical polymer chain analysis. J. Mol. Biol. 264, 919-932 (1996).

36. Khokhlov, A. R. \& Semenov, A. N. Liquid-crystalline ordering in the solution of long persistent chains. Physica A 108, 546-556 (1981).

37. Khokhlov, A. R. \& Semenov, A. N. Liquid-crystalline ordering in the solution of partially flexible macromolecules. Physica A 112, 605-614 (1982).

38. Kulp, D. T. \& Herzfeld, J. Crowding-induced organization of cytoskeletal elements. III. Spontaneous bundling and sorting of self-assembled filaments with different flexibilities. Biophys. Chem. 57, 93-102 (1995).

39. Pickett, G. T. \& Schweizer, A. P. Liquid crystallinity in flexible and rigid rod polymers. J. Chem. Phys. 112, 4881-4892 (2000). 
40. Lin, S., Numasawa, N., Nose, T. \& Lin, J. Coarse-grained molecular dynamic simulations for lyotropic liquid-crystalline solutions of semiflexible rod-like molecules. Mol. Cryst. Liq. Cryst. 466, 53-76 (2007).

41. Adamcik, J. et al. Understanding amyloid aggregation by statistical analysis of atomic force microscopy images. Nature Nanotech. 5, 423-428 (2010).

42. Binks, B. P. \& Horozov, T. S. Colloidal Particles at Liquid Interfaces 11 (Cambridge Univ. Press, 2006).

43. Besseling, R., Isa, L., Weeks, E. R. \& Poon, W. C. K. Quantitative imaging of colloidal flows. Adv. Colloid Interface Sci. 146, 1-17 (2009).

44. Rappaport, S. M., Medalion, S. \& Rabin, Y. Curvature distribution of worm-like chains in two and three dimensions. Preprint at 〈arXiv:0801.3183 > (2008).

\section{Acknowledgements}

We acknowledge support by the Electron Microscopy of ETH Zurich (EMEZ), and thank A. B. Schofield for the silica tracers, and P. A. Fischer and P. A. Rühs for discussions. S.J. acknowledges financial support from ETH Zurich (ETHIIRA TH 32-1), L.I. from MCIEF-2009-252926 and the SNSF Ambizione Fellowship PZ00P2_142532/1, and I.U. from SNF (2-77002-11).

\section{Author contributions}

S.J. performed the particle tracking, AFM experiments and analysed data. L.I. performed the cryo-SEM experiments. I.U. wrote the fibril tracking and analysis algorithm. R.M. designed and directed the study. All authors contributed to the data analysis and writing of the manuscript.

\section{Additional information}

Supplementary Information accompanies this paper at http://www.nature.com/ naturecommunications

Competing financial interests: The authors claim no competing financial interests associated with this paper.

Reprints and permission information is available online at http://npg.nature.com/ reprintsandpermissions/

How to cite this article: Jordens, S. et al. Non-equilibrium nature of two-dimensional isotropic and nematic coexistence in amyloid fibrils at liquid interfaces. Nat. Commun. 4:1917 doi: 10.1038/ncomms2911 (2013). 